Anmerkungen zur Konzeption der Enquete-Kommission

\section{Stoffstrommanagement und Wettbewerb}

\author{
Auch im Rahmen von Stoffstrommanagement-Konzepten wird auf eine Fülle \\ von Maßnahmen gesetzt - frei nach dem Motto: "Alles ist erlaubt, Hauptsache \\ es nützt der Umwelt". Einige der Vorschläge, wie horizontale Kooperationen \\ oder Selbstverpflichtungen sind jedoch durchaus widersprüchlich zu beurteilen \\ - gerade auch im Hinblick auf den freien Wettbewerb.
}

$\mathrm{D}$

Von Eike Sacksofsky er Wandel der umweltpolitischen Zielsetzungen weg von der unmittelbaren Gefahrenabwehr hin zu stärker präventiven Konzepten eines nachhaltigen Wirtschaftens bringt auch eine Änderung des Instrumentariums mit sich. Das klassische Ordnungsrecht rückt in den Hintergrund gegenüber Forderungen, die Wirtschaftssubjekte müßten selbst stärker bzw. unmittelbarer in die Verantwortung genommen werden. Während ordnungsrechtliche Vorgaben für einzelne (Schad-)Stoffe für die Wirtschaftssubjekte Daten darstellen, greift die Beeinflussung von Stoffströmen wesentlich stärker in ihr privatautonomes Handeln ein. Erst dadurch werden andere Politikbereiche, zum Beispiel das Kartellrecht als Recht, das Spielregeln für die Beziehungen zwischen Unternehmen markiert, berührt.

\section{Kosten-Internalisierung und Ordnungsrecht}

Die Beeinflussung von Stoffströmen im Produktionsprozeß erfolgt in unserem Wirtschaftssystem in erster Linie über den Markt. Die unternehmerische Entscheidung über die Allokation knapper Ressourcen entsprechend den Preissignalen stellt also bereits eine Form des Stoffstrommanagements dar. Wenn ausgerechnet natïrliche Ressourcen nicht effizient verwendet, sondern oft genug hemmungslos verschwendet werden, so liegt das daran, daß hier der Preismechanismus nicht greift. Die Nutzung natürlicher Ressourcen kostet oftmals (betriebswirtschaftlich) gar nichts - oder jedenfalls zuwenig. Gehen wir davon aus, daß der Marktmechanismus zu einer effizienten Allokation führt, wenn die Preise nicht nur einzel- sondern auch gesamtwirtschaftliche Kosten widerspiegeln, erscheint es plausibel, die externen Kosten der Umweltnutzung zu internalisieren. Auch das Management natürlicher Ressourcen könnte dann dem Marktsystem überlassen werden. Daraus ergibt sich die überragende Bedeutung ökonomischer Instrumente für die Umweltpolitik.

Das Konzept des Stoffstrommanagements, das die Enquete-Kommissionzum Schutz des Menschen und der Umwelt in ihrem entsprechenden Bericht (1) entwickelte, orientiert sich eng am Marktsystem. Auch die Kommission geht davon aus, daß Umweltziele dann verfehlt werden, wenn „die Preise nicht die ökologische Wahrheit sagen". Idealerweise, so die Kommission, müßten daher externe Effekte in die Marktpreise internalisiert werden.

Dies ist aber nicht immer realistisch; gerade dann, wenn die zu internalisierenden Kosten nicht exakt zu beziffern sind oder selbst auf Werturteilen, zum Beispiel auf Zeitpräferenzen, beruhen. In diesem Fall können ordnungsrechtliche Instrumente - außer zur Gefahrenabwehr - eine wichtige Rolle spielen. Die Quotenvorgaben der Verpackungsverordnung, ohne die jeder Zwang zu umweltschonendem Verhalten fehlte, sind ein Beispiel einer solchen second-best Strategie (2).

Die Enquete-Kommission gibt sich damit nicht zufrieden, sondern macht sich auf die Suche nach weiteren Handlungsmöglichkeiten - den informatorischen und freiwilligen Maßnahmen. Aus wettbewerblicher Sicht ist zu fragen, inwieweit diese Strategien mit dem Marktmechanismus kompatibel sind - sich also die Steuerungsfunktion des Wettbewerbs zunutze machen oder sie jedenfalls nicht konterkarieren. Nur dann werden sie beanspruchen können, zur Allokationseffizienz beizutragen.

\section{- Freiwillige Maßnahmen - individuelle Strategien}

Das Spektrum freiwilliger Maßnahmen ist denkbar weit. Handelt es sich dabei um einzelbetriebliche Maßnahmen, sind ihre Wettbewerbswirkungen neutral. In der Regel werden sie zumeist sogenannte no-regrets-Strategien sein, also Maßnahmen, die dem Gewinnmaximierungskalkül nicht zuwiderlaufen (3). Zunehmend wird Umweltverträglichkeit von Produktion oder Produkten auch als Wettbewerbsparameter eingesetzt. Das funktioniert in erster Linie dann, wenn sich ein Anbieter dank eines Wettbewerbsvorstosses von den übrigen Wettbewerbern seiner Branche absetzen kann.

Interessanter sind - aus wettbewerblicher Sicht - Kooperationsstrategien. Kooperationen widersprechen dem grundlegenden Steuerungsprinzip von Marktwirtschaften, der Konkurrenz. Der Wettbewerbspolitiker wird ihnen daher zunächst mit Skepsis begegnen. Demgegenüber zeichnet die Enquete-Kommission ein eher positives Bild von Kooperationen. Zwar heißt es, „daß Kooperationen in einer auf Konkurrenz und Arbeitsteilung ausgerichteten Gesellschaft nicht der Regelfall sind, sondern in einem Spannungsverhältnis zu marktwirtschaftlichen Mechanismen stehen". Eine Seite weiter erfahren wir dann aber, daß das Kooperationsprinzip in der Umweltpolitik theoretisch einen hohen Stellenwert habe. Und weiter: „Insbesondere Stoffpolitik und Stoffstrommanagement (...) müssen auf motivationsfördernde, flexible und effiziente Instrumente setzen, bei denen Kommunikation, ökonomische Anreize und Kooperationen im Vordergrund stehen".

\section{Horizontale und vertikale Kooperation}

Versucht man sich dem Phänomen von Kooperationen zu nähern, bietet sich zunächst die klassische Unterscheidung zwischen horizontalen und vertikalen Kooperationen an.

Vertikale Kooperationen sind solche zwischen Lieferant und Abnehmer, also zwischen Unternehmen, die nicht in einem unmittelbaren Wettbewerbsverhältnis stehen. Sie kommen in der Regel zur Anwendung, wenn Probleme angesprochen sind, die auf einer Wertschöpfungsstufe nicht lösbar sind. Das liegt bei der Betrachtung von Stoffströmen ,von der Wiege bis zur Bahre" natürlich nahe. Dieser Zusammenarbeit auf unterschiedlichen Stufen der Wertschöp- 
fungskette, also entlang eines Stoffstroms, steht zunächst nichts entgegen. Genauso wie in der Automobilindustrie zwischen Zulieferer und Hersteller eine enge Zusammenarbeit zur Optimierung von Produktionsabläufen stattindet, könnte man sich eine Kooperation zur gemeinsamen Erreichung von Umweltzielen vorstellen. Ein wettbewerbliches Problem entsteht dabei nur dann, wenn der eine Kooperationspartner infolge von Marktmacht dem anderen seine Bedingungen aufzwingen kann, oder ein marktumspannendes Netz gleichartiger Vertikalverträge entsteht. Entsprechend sind Vertikalabreden nach dem deutschen Kartellrecht grundsätzlich erlaubt und können nur im Wege der Mißbrauchsaufsicht angegriffen werden, wenn Marktmacht im Spiel ist.

Von ganz anderem Kaliber ist die horizontale Kooperation zwischen Wettbewerbern, wie sie zum Beispiel Branchenabkommen darstellen. In der ökonomischen Diskussion wird sie auch mit so häßlichen Worten wie Kartell belegt. Denn Absprachen zwischen Wettbewerbern sind in verschiedener Hinsicht problematisch. Vor allen Dingen können sie entgegen jeglichem Verursacherprinzip dazu dienen, die Kosten des Umweltschutzes gemeinsam auf Dritte abzuwälzen.

\section{- Horizontale Kooperationen - ein Blick auf die Praxis}

Werfen wir daher einen etwas genaueren Blick auf einige Kooperationspläne der jüngeren Zeit. Ende vergangenen Jahres einigten sich unter dem sanften Druck des Umweltministeriums Handel, Getränkeabfiuller und Behälterindustrie, auf jede Getränkebüchse einen „Dosengroschen“ auf den Verkaufspreis aufzuschlagen. Damit, so das scheinbar hehre Ziel, hätte der Vormarsch der Einwegverpackung zu Lasten der Mehrweggebinde gebremst werden können. Nebenbei wären auch noch ein paar Mark zur Bekämpfung des Littering, also zur Reinigung der Landschaft von achtlos weggeworfenen Dosen, abgefallen. Wer wollte solch edlem Ansinnen widersprechen.

Ein Blick auf die Fakten dieser freiwilligen Kooperation spricht jedoch einen andere Sprache. Bei einem Absatz von vier bis fünf Milliarden Dosen im Jahr wären ceteris paribus unter sonst gleichen Umständen - 400 bis 500 Millionen Mark in die Taschen des Kartells geflossen. Davon waren etwa 100 Millionen Mark zur Bekämpfung des Littering verplant.
Der Restbetrag wäre einer privaten Steuer gleich bei der sich absprechenden Industrie verblieben. Sicher wäre, abhängig von der Preiselastizität der Nachfrage, der Dosenabsatz infolge der Preiserhöhung zurückgegangen und der effektive Betrag geringer ausgefallen. Darauf kommt es aber im Hinblick auf das prinzipielle Problem nicht an.

Ein anderes Beispiel ist die Debatte um eine Neuregelung der Altautoentsorgung. Nachdem die Industrie - nach langem Hin und Her akzeptiert hatte, Altautos kostenlos zurückzunehmen, legte sie den Entwurf einer Selbstverpflichtung vor, der vorsah, daß Autos nur bis zu einem Alter von zwölf Jahren kostenlos zurïckgenommen würden, soweit sie ausschließlich von Vertragswerkstätten gewartet wurden und dabei nur Originalersatzteile verwendet wurden. Auch dieses Konzept war mit dem Kartellrecht nicht vereinbar, insbesondere weil es die freien Werkstätten und den freien Teilehandel benachteiligt hätte. Aus ökologischer Sicht ist anzumerken, daß damit offensichtlich ein Anreiz zur Verkürzung der Produktlebenszyklen gesetzt werden sollte, um den Neuwagenverkauf anzukurbeln.

\section{- Negative Sanktionierung - Negativer Anreiz}

Instruktiv ist auch die Frage der Entsorgung von Altbatterien. In Umsetzung einer entsprechenden EG-Richtlinie gibt es bereits seit längerem ein funktionierendes System der Erfassung und Verwertung schadstoffhaltiger Gerätebatterien. Im Rahmen der „Zweiten freiwilligen Selbstverpflichtung" von Batterieindustrie und Handel wird nun vorgeschlagen, auch schadstoffarme und schadstoffreie Batterien, die etwa 85 Prozent aller Gerätebatterien ausmachen, im Wege eines kollektiven Systems zu erfassen und zu verwerten. Im Unterschied zu schadstoffhaltigen Batterien, die sich einigermaßen kostendeckend verwerten lassen, schlagen die Erfassungs- und Verwertungskosten bei schadstoffarmen und -freien Batterien sehr viel mehr zu Buche. Eine Realisierung der geplanten Selbstverpflichtung würde die Herstellerabgabepreise um bis zu 68 Prozent erhöhen.

Die Vergemeinschaftung eines derart großen Preisbestandteils würde im Ergebnis nichts anderes bedeuten, als über die Beschränkung des Preiswettbewerbs zwischen den Batterieherstellern die Weitergabe der Kosten an den Endverbraucher sicherzustellen. Zusätzlich würden durch diese Zusatzbelastung auf alle Batterien, die erfolgreichen und ökologisch erstrebenswerten Anstrengungen der Industrie, schadstoffhaltige durch schadstoffarme oder -freie Batterien $\mathrm{zu}$ ersetzen, negativ sanktioniert. Ökonomisch würde damit ein negativer Anreizeffekt gesetzt, der sich gegen sinnvolle Innovation auswirkt. Ein weiteres ist interessant. Mit der Umsetzung der Selbstverpflichtung entstïnde ein Nachfragekartell aller Batteriehersteller nach Erfassungs- und Verwertungsleistungen. Ein Innovationswettbewerb um ökonomisch oder ökologisch bessere Verwertungsverfahren fände nicht mehr statt.

\section{- Weiche Maßnahmen oder harte Kartelle?}

Diese Beispiele machen einige Grundprobleme deutlich, die horizontale Absprachen im Umweltbereich mit sich bringen:

Freiwilligen Selbstverpflichtungen, die gegen Gesetz gegen Wettbewerbsbeschränkungen (GWB) verstoßen, ist die Unverbindlichkeit der Vereinbarungen und das Fehlen von Sanktionsmechanismen immanent. Denn Verträge zwischen Wettbewerbern, die den Tatbestand des Kartellverbots ( 1 GWB) erfüllen, sind kraft Gesetz unwirksam. Daraus ergibt sich zwingend, daß Absprachen über Sanktionen im Kartell, auch wenn sie getroffen werden, zivilrechtlich nicht durchsetzbar sind. Zweierlei kann daraus resultieren:

Die Beteiligten einigen sich auf eine Absprache, die niemandem weh tut. Dann entfält das Sanktionsproblem, weil das Mitmachen nichts kostet. Ökonomisch bleibt eine solche Kooperation folgenlos, ökologisch bringt sie dann freilich nichts. Dieser Fall ist nicht so exotisch, wie er klingen mag. Ein Beispiel stellt die Selbstverpflichtung der Arbeitsgemeinschaft Graphische Papiere (AGRAPA) zur Wiederverwertung ihrer Produkte dar. Um die vom Umweltministerium vorgelegte Altpapierverordnung abzuwenden, boten die beteiligten Unternehmen an, freiwillig die Wiederverwertungsquoten für graphische Papiere von derzeit 53 auf 60 Prozent im Jahr $2000 \mathrm{zu}$ erhöhen. Nachdem das Bundeskartellamt Bedenken gegen diese Verpflichtung angemeldet hatte, legte die AGRAPA überzeugend dar, daß diese Quoten sich bei ganz normaler Marktentwicklung einstellen würden, ohne daß es irgendeiner zusätzlichen Anstrengung der beteiligten Unternehmen bedïrfte (4). 


\section{Keine Garantie auf Effizienz}

Die zweite Möglichkeit ist, daß den Unternehmen durch eine entsprechende Verpflichtung tatsächlich Kosten entstehen. Das Risiko von Trittbrettfahrerverhalten und damit die Ausbeutung ehrlicher Unternehmen durch free rider, die sich nicht an die Vereinbarung halten, ist dementsprechend hoch. Um diese Probleme zu umgehen, besteht ein beträchtlicher Anreiz für die Branche, eine Absprache zu Lasten Dritter zu treffen. Insoweit kann auch nicht davon gesprochen werden, Selbstverpflichtungen seien regelmäßig ökonomisch effizient. Die Kommission meint dies aus der Tatsache der Freiwilligkeit der Teilnahme aus niedrigen Transaktionskosten folgern zu können (5).

Branchenabkommen verhindern gerade, daß sich ein Preissignal für die effiziente Nutzung von Umweltressourcen herausbildet. Ein Preisaufschlag im Kartell verbessert zwar die Kosten-Erlös-Relation der beteiligten Unternehmen, ob damit aber eine echte Leistungssteigerung einhergeht, ist völlig offen. Ein unlängst erschienenes Gutachten im Auftrag des Bundesministeriums für Wirtschaft zu Möglichkeiten und Grenzen von freiwilligen Umweltschutzmaßnahmen der Wirtschaft unter ordnungspolitischen Aspekten schätzt die Effizienzwirkungen eher gering ein '(6). Dabei eröffnet das deutsche Kartellgesetz die Möglichkeit der Legalisierung von Kartellabsprachen unter bestimmten Voraussetzungen ( $\S 2-8 \mathrm{GWB}$ ). Von besonderer Bedeutung, auch quantitativ, ist die Legalisierung zum Zwecke der Rationalisierung ( $\$ 5$ Abs. 2, 3; $\S 5$ b). Soweit Umweltkartelle diese Voraussetzungen erfuillen, stehen ihnen diese Legalisierungsmöglichkeiten selbstverständlich offen. Die angesprochenen Beispiele erfüllen sie aber gerade nicht.

\section{Ordnungsrecht vonnöten}

Ein Vorteil von Kooperationslösungen wird auch in der Hoffnung gesehen, sie könnten schneller und flexibler umgesetzt werden als staatliche Maßnahmen. Besonders begründet ist sie nicht. Es besteht weitgehende Einigkeit, daß freiwillige Maßnahmen, die staatliches Handeln ersetzen sollen, nur dann wirken, wenn das Drohpotential des Ordnungsrechts verfügbar ist. Um den zeitlichen Vorlauf, rechtliche Instrumente vorzubereiten, mit denen dann gedroht werden kann, kommt man also nicht herum. Auch die praktischen Erfahrungen mit den oben genannten Beispielen zeigen, daß sich mit freiwilligen Maß- nahmen ausgezeichnet auf Zeit spielen läßt. Einen grundsätzlichen Vorteil freiwilliger Kooperationen sieht die Enquete-Kommission schließlich darin, daß den Unternehmen ihr individueller Handlungsspielraum erhalten bleibe. Das wäre nur dann richtig, wenn die Absprache sich allein auf das Ergebnis des Handelns erstreckte, ohne jede Übereinkunft über ihre Durchführung. Eine solche, völlig unverbindliche Absprache ist wenig wahrscheinlich. Auch scheint fraglich, ob sich staatliche Umweltpolitik damit zufriedengeben könnte. Die Erfahrungen mit Kooperationsmodellen legen eher nahe, daß sich Unternehmen nicht nur über das Ziel, sondern auch recht konkret über den Weg dahin absprechen, also Durchführungsvereinbarungen treffen. Dann aber bleibt kein Raum mehr für individuelle Strategien, vielmehr wird der Innovationswettbewerb um neue Formen der Zielerreichung ausgeschaltet, weil sich die ganze Branche auf einen Weg verständigt hat.

\section{Eine Schlußbemerkung}

Stoffstrommanagement und freiwillige Selbstverpflichtungen sind nicht identisch. Stoffstrommanagement besteht aus einem breiten Maßnahmenbündel, von dem Selbstverpflichțungen nur einen kleinen Ausschnitt darstellen. Wenn horizontale Kooperationen und Selbstverpflichtungen dennoch in den Mittelpunkt dieses Beitrags gestellt wurden, dann deshalb, weil sich an ihnen exemplarisch aufzeigen läßt, wo die Problematik einer Umweltpolitik liegt, die nach dem Motto verfährt: „Alles ist erlaubt, Hauptsache, es nützt der Umwelt". Das passiert auch in der Praxis. Von verschiedenen Unternehmensverbänden über einzelne Gewerkschaften bis hin zum Bundesministerium für Umwelt erschallt der gemeinsame Ruf, das Kartellrecht im Interesse des Umweltschutzes zu lockern, also Umweltkartelle ausdriicklich zu erlauben. Überspitzt ausgedruickt steht dahinter die Vorstellung, Umweltpolitik werde umso besser und erfolgreicher, je mehr Instrumente zum Einsatz kommen - und seien sie noch so widersprüchlich. Man mag ja darüber diskutieren, ob der Wettbewerb und das Marktsystem eine vorteilhafte Organisation der Wirtschaft darstellen. Nur wenn man, wie es die Enquete-Kommission ausdrücklich tut, davon ausgeht, daß ein Preissystem (das Externalitäten internalisiert hat) ein überlegener Steuerungsmechanismus gegenüber zentral geplanten oder korporatistischen Modellen ist, dann ist das positive Bild von
Kooperationslösungen inkonsequent. Vielmehr sollten sie daran gemessen werden, inwieweit sie den Organisationsprinzipien unserer Wirtschaft Rechnung tragen. Das deutsche Kartellrecht, das zugegeben Umweltkartellen oft genug im Weg steht, ist dafür ein guter Referenzrahmen.

\section{Anmerkung}

Der Beitrag gibt allein die Meinung des Autors wieder und ist keine offizielle Stellungnahme des Bundeskartellamtes.

\section{Liferatur}

1) Bericht der Enquete-Kommission "Schutz des Menschen und der Umwelt: Die Industriegesellschaft gestalten.

2) Monopolkommission, 11. Hauptgutachten 1994/95, Tz. 89

3) Beispiele dazu in Führ, ZfU 4/1994, S.445 ff.

4) vgl. Tätigkeitsbericht des Bundeskartellamtes

1993/94, Bundestagsdrucksache 13/1660, S. 127.

5) vgl. 4) $S .317$

6) vgl. Bergmann/Brockmann/Rennings (Zentrum für Europäische Wirtschaftsfoschung): Möglichkeiten und Grenzen von freiwilligen Umweltschutzmaßnahmen der Wirtschaft unter ordnungspolitischen Aspekten. Mannheim, Mai 1996

\section{Der Autor}

Diplom-Volkswirt Eike Socksatsky ist Mitarbeiter der Grundsatzabieilung des Bundeskariellamtes. Kontakt: Bundeskariellamt, Mehringdamm 129, 10965 Berlin, Tel. (030) $69580-0$

\section{Ökologisches Wirtschaften}

\section{Was Sie noch bestellen können:}

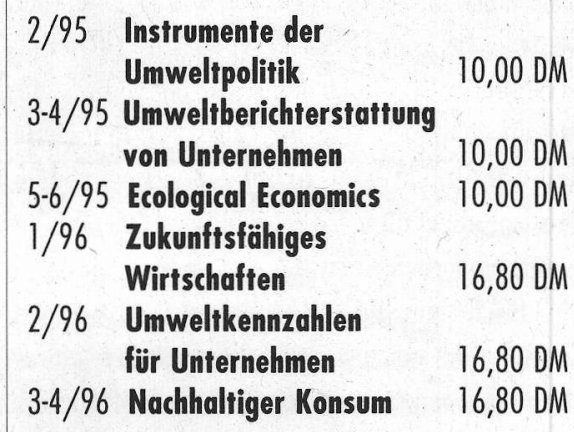

zu bestellen bei:

ökom GmbH, Waltherstr. 29 Rckgb., 80337 München

Tel. (089) 544 184-0, Fax -99 
(c) 20I0 Authors; licensee IÖW and oekom verlag. This is an article distributed under the terms of the Creative Commons Attribution Non-Commercial No Derivates License (http://creativecommons.org/licenses/by-nc-nd/3.o/), which permits unrestricted use, distribution, and reproduction in any medium, provided the original work is properly cited. 